\title{
Collective excitations in asymmetric parabolic quantum wells
}

\author{
P. I. Tamborenea and S. Das Sarma \\ Department of Physics \\ University of Maryland \\ College Park, Maryland 20742
}

PACS Code: 73.20.Mf, 71.45.Gm, 78.20.Ls, 78.66.Fd

\begin{abstract}
We study within the linear response theory the far-infrared optical absorption spectrum and the collective excitations of an asymmetric, wide parabolic $A l_{x} G a_{1-x}$ As quantum well, consisting of two half-parabolas of different curvatures, in the presence of an in-plane magnetic field. We employ a self-consistent-field approach in the local-density approximation using parameter values of a recent experimental study. At low $N_{s}$, there is only one resonance corresponding to the lowest inter-Landau-level transition, and at high $N_{s}$ we obtain two resonances at frequencies corresponding to the different curvatures of the well, in good agreement with experiment. We also calculate the optical spectra without any magnetic field and find similar trends as a function of $N_{s}$, i.e., one resonance at low $N_{s}$ and two main resonances at high $N_{s}$.
\end{abstract}


Remotely doped parabolic quantum wells (PQW) show infrared magneto-optical spectra [1, 2, 3] in agreement with the generalized Kohn theorem [4], which states that an electron gas in a perfect parabolic confinement and with a magnetic field applied in an arbitrary direction, absorbs long-wavelength light only at the two frequencies that correspond to excitations in the center of mass (CM) motion - the so-called "Kohn modes". For a number of confining potentials with deviations from perfect parabolicity, both experiment [3, 5] and theory [6, 7, 8] show that the CM modes are robust against the nonparabolicities (although their energies may be slightly shifted), and that additional excitations, usually with small oscillator strength, appear in the far-infrared (FIR) optical spectra. In the presence of an in-plane magnetic field the changes in the spectra induced by nonparabolicities may be more dramatic, as shown for an overfilled PQW [9], where the CM mode, which in the perfect parabolic case is separated from the continua of inter-Landau-level transitions, is broadened into a continuum.

Recently [10, the FIR optical absorption spectra of an asymmetric PQW consisting of two half parabolas of different curvatures have been measured in the presence of an inplane magnetic field $\vec{B}$, with normally incident radiation linearly polarized with $\vec{E}^{R A D} \perp \vec{B}$. For a perfect PQW (given by $V(z)=\alpha_{0} z^{2}$ ) in this Voigt geometry, the generalized Kohn theorem predicts a unique absorption frequency $\omega=\left(\omega_{0}^{2}+\omega_{c}^{2}\right)^{1 / 2}$, where $\omega_{0}=\left(2 \alpha_{0} / m^{*}\right)^{1 / 2}$ is the frequency of the harmonic-oscillator confining potential with $m^{*}$ being the electron effective mass in GaAs, and $\omega_{c}=\left(e B / m^{*} c\right)^{1 / 2}$ is the cyclotron frequency. In the perfect parabolic case this resonance is expected to be independent of the electron sheet density $N_{s}$, as corroborated by experiment [2, 3]. For the asymmetric PQW, instead, the generalized Kohn theorem is no longer valid, and the FIR spectra are seen to depend on $N_{s}$. For large $N_{s}$ there are two resonances corresponding to the two curvatures in the well, and as $N_{s}$ is decreased they merge into a unique resonance corresponding to the lowest inter-Landaulevel transition.

In this paper, we present results of a self-consistent quantum mechanical calculation of optical spectra and collective excitations for the asymmetric PQW experimentally studied in Ref. [10], both with a magnetic field in the Voigt geometry and without a magnetic field. With an applied magnetic field, in the limits of low and high $N_{s}$ we obtain excellent 
agreement with the experimental results, and we calculate the induced density fluctuations associated with the resonances observed at high $N_{s}$. With $\vec{B}=0$ we obtain a similar behavior in the FIR spectra as a function of $N_{s}$, with one resonance at low $N_{s}$ and two resonances at the Kohn frequencies at high $N_{s}$.

Intersubband optical absorption for a quasi-2D electron gas in the presence of an inplane magnetic field, with the radiation polarized in the growth direction has been calculated by Ando [11, 12], and his treatment was adapted to the normal incidence case by Dempsey and Halperin [9]. In this paper we use a similar self-consistent-field approach, which includes exchange and correlations effects within the Local Density Approximation (LDA).

We choose the growth axis in the z-direction, the magnetic field $\vec{B}=(0, B, 0)$ and the gauge $\vec{A}=(B z, 0,0)$. Ignoring the Zeeman energy, the effective single-particle Hamiltonian is 9 , 13.

$$
H=\frac{\left(p_{x}+m^{*} \omega_{c} z\right)^{2}}{2 m^{*}}+\frac{p_{y}^{2}}{2 m^{*}}+\frac{p_{z}^{2}}{2 m^{*}}+V_{\mathrm{eff}}(z)
$$

where $V_{\text {eff }}(z)=V_{\text {conf }}+V_{H}(z)+V_{\text {xc }}(z)$ is the sum of the confining $V_{\text {conf }}(z)$, Hartree $V_{H}$, and exchange-correlation $V_{x c}$ 14 potentials. The eigenfunctions of $H$ can be factorized as

$$
\psi_{n k_{x} k_{y}}(x, y, z)=\frac{e^{i k_{x} x}}{L_{x}^{1 / 2}} \frac{e^{i k_{y} y}}{L_{y}^{1 / 2}} \varphi_{n k_{x}}(z)
$$

and the corresponding eigenenergies are $E_{n}\left(k_{x}, k_{y}\right)=\frac{\hbar^{2} k_{y}^{2}}{2 m^{*}}+\varepsilon_{n}\left(k_{x}\right)$. In this work, the quasi-continuous set of values of the quantum number $k_{x}$ obtained when periodic boundary conditions are applied in the $\mathrm{x}$-direction is discretized into a coarser mesh to make the problem tractable numerically [12, 9]. The electron density is then calculated as

$$
n(z)=\sum_{n k_{x}} N_{n k_{x}}\left|\varphi_{n k_{x}}(z)\right|^{2}
$$

where $N_{n k_{x}}$ is given, at zero temperature, by 


$$
N_{n k_{x}}=\frac{2}{\pi L_{x}}\left(\frac{2 m^{*}}{\hbar^{2}}\right)^{1 / 2} \sum_{n k_{x}} \theta\left(E_{F}-\varepsilon_{n}\left(k_{x}\right)\right)\left(E_{F}-\varepsilon_{n}\left(k_{x}\right)\right)^{1 / 2},
$$

where $E_{F}$ is the Fermi energy and $\theta$ is the unit step function. The self-consistent singleparticle problem without magnetic field is solved following a method analogous to the one just described [6, 8]. Having solved the self-consistent single-particle problem we proceed to calculate the absorption spectrum. In the Voigt geometry, the absorbed power is proportional to the real part of the xx-component of the modified two dimensional conductivity tensor, which is related to the zz-component by [9] $\operatorname{Re}\left[\tilde{\sigma}_{x x}^{2 D}\right]=\frac{\omega_{c}^{2}}{\omega^{2}} \operatorname{Re}\left[\tilde{\sigma}_{z z}^{2 D}\right]$. In our calculations with magnetic field we obtain $\tilde{\sigma}_{z z}^{2 D}$ following the method described in Refs. [12, 9], and for the zero magnetic field calculations we follow Refs. [15, 6, 8]. In both cases one obtains

$$
\tilde{\sigma}_{z z}^{2 D}=-i \omega e^{2} \sum_{\eta} \frac{f_{\eta}^{(z)}}{\tilde{\varepsilon}_{\eta}^{2}-(\hbar \omega)^{2}-2 i \hbar^{2} \omega / \tau},
$$

where $f_{\eta}^{(z)}$ are the oscillator strengths corresponding to the resonant energies $\tilde{\varepsilon}_{\eta}$, which include the depolarization and the exciton-like corrections [12, 15], and $\tau$ is a phenomenological relaxation time. In the Voigt geometry problem the index $\eta$ denotes a discretized quasi-continuous variable whereas in the zero magnetic field case it is simply an integer variable. This implies that at zero magnetic field the absorption spectrum consists of a set of isolated resonant frequencies, and with an in-plane magnetic field there can be, in principle, both isolated and broad quasi-continuum resonances, which is indeed the case for the asymmetric parabolic well under consideration.

Our model potential describing the asymmetric PQW sample studied in Ref. [10 is shown in Fig. 1, and consists of two half-parabolas with curvatures $\alpha_{1}=5.1 \times 10^{-5} \mathrm{meV} / \AA^{2}$ and $\alpha_{2}=6.2 \times 10^{-5} \mathrm{meV} / \AA^{2}$, and it is $3000 \AA$ wide. For the calculations in the Voigt geometry, we choose a magnetic field in the region of the observed resonances of $B=5.8 T$. In Fig. 1 we show the calculated self-consistent potentials for some values of the sheet density $N_{s}$, which, as expected, become flattened over a wider region in the center of the well, as the electron slab width increases with $N_{s}$. Note that for the values of $N_{s}$ considered in the experiment, and in our calculations with magnetic field, only the lowest subband is 
occupied at zero temperature. The electron density profiles $n(z)$ are shown as an inset in Fig. 1. We checked that the abrupt change in the curvature at $z=0$ does not produce unphysical results by using a similar potential with a graded change in curvature, and the density profile was identical to the original one within our numerical precision.

The optical absorption spectra for the asymmetric quantum well and for several densities $N_{s}$ in the range used in Ref. [10] are shown in Fig. 2. The frequencies associated with the half-parabolas of curvatures $\alpha_{1,2}$ are $\omega_{1,2}=2 \alpha_{1,2} / m^{*}$. The frequencies $w_{K 1, K 2}$ marked for reference on each panel of Fig. 2 are the "Kohn" frequencies of each half-parabola, $w_{K 1, K 2}=$ $\left(\omega_{1,2}^{2}+\omega_{c}^{2}\right)^{1 / 2}$ - the only frequencies at which perfect parabolic wells with curvatures $\alpha_{1,2}$ would absorb long-wavelength radiation in the Voigt geometry. The phenomenological scattering time $\tau=20 \times 10^{-12} s$, taken to be a constant in our calculations, is taken from the linewidth of the narrower resonance in the experiment 10. For a perfect PQW the generalized Kohn theorem predicts a unique resonance independent of the electron density. In our case, in contrast, we obtain a strong dependence on $N_{s}$. For small $N_{s} \lesssim$ $4.7 \times 10^{10} \mathrm{~cm}^{-2}$ there is only one resonance (for example, $\tilde{\varepsilon}=10.644 \mathrm{meV}$ at $N_{s}=$ $0.1 \times 10^{10} \mathrm{~cm}^{-2}$ ), corresponding to the optical transition between the first two energy levels of the bare confining potential in the presence of the in-plane magnetic field, with an energy $\varepsilon_{1}\left(k_{x}=0\right)-\varepsilon_{0}\left(k_{x}=0\right)=10.648 \mathrm{meV}$. As $N_{s}$ increases another resonance appears at an energy lower than $\omega_{K 1}$, and gradually moves to $\omega_{K 1}$ while the other resonance also shifts to end up at $\omega_{K 2}$ for high $N_{s}$. At a sheet density $N_{s}=7.5 \times 10^{10} \mathrm{~cm}^{-2}$ (the highest $N_{s}$ reported in the experiment) we obtain two resonances $\omega_{1,2}^{R E S}$ very close to the "Kohn" frequencies $\omega_{K 1, K 2}$. We note that a direct comparison of the theoretical and calculated spectra is not possible because the experimental results correspond to a magnetic field sweep at a constant resonance frequency whereas the calculated optical spectra are given as a function of frequency at a fixed magnetic field. In order to make a quantitative comparison with experiment, we use the resonant frequencies $\omega_{1,2}^{R E S}$ to compute the harmonic oscillator frequencies of the well halves $\omega_{1,2}^{C A L C}=\left(\left(\omega_{1,2}^{R E S}\right)^{2}-\omega_{c}^{2}\right)^{1 / 2}$, and obtain their difference $\omega_{2}^{C A L C}-\omega_{1}^{C A L C}=0.378 \mathrm{meV}$. This shows an $8 \%$ discrepancy with the input value $\omega_{2}-\omega_{1}=0.350 \mathrm{meV}\left(\omega_{1,2}\right.$ are the values used in the definition of the confining potential) which is comparable to the $4 \%$ discrepancy found in experiment at the same 
sheet density. Therefore, we obtain a very good agreement with the experimental results of Ref. 10 for low and high $N_{s}$. The agreement at high $N_{s}$ also confirms the strong magnetic field classical arguments based on the Magarill-Chaplik [16] theory given in Ref. [10]. At intermediate $N_{s}$, however, the position of the weaker resonance seems to evolve in different ways as a function of $N_{s}$ in theory and experiment. In the experiment, the two "Kohn" resonances found at high $N_{s}$ appear to merge into one absorption peak as $N_{s}$ is reduced, in contrast to the separation and vanishing of one of the resonances found in our calculation. One possibility is that this discrepancy appears as a result of the different type of spectra obtained in theory and experiment (frequency versus magnetic field sweep) and then an improved fit to the experimental results at intermediate $N_{s}$ could be obtained if a magnetic field and density dependent scattering time $\tau\left(B, N_{s}\right)$ is included in the calculation.

Now we comment on two aspects of the collective excitations of the electron gas in the asymmetric parabolic well in the presence of an in-plane magnetic field. The CM mode predicted by the generalized Kohn theorem for a perfect parabolic confinement is a rigid oscillatory motion of the electron slab. In linear response, its density fluctuations $\delta n(z)$ are proportional to $d n(z) / d z$ [9]. In Fig. 3 we show the fluctuation profiles $\delta n(z)$ for the resonant modes at $N_{s}=7.5 \times 10^{10} \mathrm{~cm}^{-2}$ together with the derivative $d n(z) / d z$. We see that the resonant modes very accurately describe rigid translations of the electron gas in the corresponding halves of the well — right and left halves for the resonances with $\omega_{K 1}$ and $\omega_{K 2}$, respectively. Finally, a notable difference between our results for the asymmetric PQW of Ref. [10] and a previous study of an overfilled PQW in the Voigt geometry [9] is the interplay between the main resonances or "Kohn modes" and the continuum of inter-Landau-level transitions. For the overfilled PQW the CM mode is broadened into a continuum, whereas for the asymmetric PQW the "Kohn modes" of the two half parabolas are split off from the continuum of inter-Landau-levels at high densities, and at lower densities one of them becomes a broad continuum resonance while the other one remains an isolated resonance as in the case of perfect parabolic confinement.

Figure 4 shows the calculated IR optical spectra for the asymmetric PQW without magnetic field for $N_{s}$ in the range $0.1-1.6 \times 10^{11} \mathrm{~cm}^{-2}$. The number of occupied subbands goes from 1 to 4, and we keep 10 subbands in the calculation of the optical spectra. 
Again, for low $N_{s}$ there is only one resonance corresponding to the lowest optical transition

of the bare well, and at high $N_{s}$ there are two resonances close to the frequencies $\omega_{1}$ and $\omega_{2}$. A surprising feature is the appearance of two resonances of similar weight at $N_{s}=0.6 \times 10^{11} \mathrm{~cm}^{-2}$, when the second subband is populated, which disappears at higher $N_{s}$.

In conclusion, we have studied the FIR optical absorption spectra of an asymmetric PQW with a magnetic field in the Voigt geometry and without a magnetic field using the self-consistent LDA approach. We compare our magnetic field results with experimental spectra and find good quantitative agreement with experiment for low and high $N_{s}$ and quantitative differences in the spectra at intermediate $N_{s}$. The origin of this disagreement remains an open question. In particular, experiments with a frequency sweep at constant magnetic field would permit a more direct comparison between theory an experiment. At zero magnetic field, the general trends of the spectra are similar to those of the Voigt geometry results, and here again an experimental measurement of the optical spectra would be useful to compare the accuracy of the LDA approximation with and without magnetic field.

The authors are grateful to I. K. Marmorkos, Y. K. Hu, K. Karrai, C. Stafford, and especially to H. D. Drew for useful discussions. This work is supported by the US-ARO, US-ONR, and the US-DoD.

\section{References}

[1] S. Das Sarma, in Topics in Condensed Matter Physics, edited by M. Das (Nova Science, New York, 1993).

[2] K. Karrai, H. D. Drew, M. W. Lee, and M. Shayegan, Phys. Rev. B39, 1426 (1989); K. Karrai, X. Ying, H. D. Drew, and M. Shayegan, Phys. Rev. B40, 12020 (1989).

[3] A. Wixforth, M. Sundaram, J. H. English, and A. C. Gossard, in Proceedings of the 20th International Conference on the Physics of Semiconductors, edited by E. M. Anastassakis and J. D. Joannopoulos (World Scientific, Singapore, 1990), p. 1705. 
[4] L. Brey, N. F. Johnson, and B. Halperin, Phys. Rev. B40, 10647 (1989).

[5] K. Karrai, X. Ying, H. D. Drew, M. Santos, M. Shayegan, S.-R. E. Yang, and A. H. Mac Donald, Phys. Rev. Lett. 67, 3428 (1991).

[6] L. Brey, Jed Dempsey, N. F. Johnson, and B. I. Halperin, Phys. Rev. B42, 1240 (1990).

[7] Jed Dempsey, and B. I. Halperin, Phys. Rev. B45, 3902 (1992); 47, 4662 (1993).

[8] M. P. Stopa and S. Das Sarma, Phys. Rev. B, 45, 8526 (1992).

[9] Jed Dempsey, and B. I. Halperin, Phys. Rev. B47, 4674 (1993).

[10] X. Ying, K. Karrai, H. D. Drew, M. Santos and M. Shayegan, Phys. Rev. B46, 1823 (1992).

[11] T. Ando, J. Phys. Soc. Jpn. 39, 411 (1975).

[12] T. Ando, J. Phys. Soc. Jpn. 44, 475 (1978).

[13] M. P. Stopa and S. Das Sarma, Phys. Rev. B, 47, 2122 (1993).

[14] F. Stern and S. Das Sarma, Phys. Rev. B, 30, 840 (1984).

[15] T. Ando, Z. Phys. B26, 263 (1977).

[16] L. I. Magarill and A. V. Chaplik, Pis'ma Zh. Eksp. Teor. Fiz. 40, 301 (1984) [JETP Lett. 40, 1089 (1984)].

\section{Figure Captions}

Figure 1

Model bare potential of the asymmetric parabolic well (solid line), and calculated selfconsistent potentials with an in-plane magnetic field of $B=5.8 T$, for $N_{s}=2.4$ (dash line), 4.7 (dotted line), and $7.5 \times 10^{10} \mathrm{~cm}^{-2}$ (dash-dot line); inset: corresponding calculated self-consistent densities.

Figure 2 
Calculated absorption spectra for the asymmetric parabolic quantum well with a magnetic field $B=5.8 \mathrm{~T}$ in the Voigt geometry for various sheet densities $N_{s}$ (given in units of $\left.10^{10} \mathrm{~cm}^{-2}\right) . \omega_{K 1}$ and $\omega_{K 2}$ are the "Kohn" frequencies associated with the curvatures of the two half-parabolas of the well.

\section{Figure 3}

Density fluctuations $\delta n(z)$ (dotted lines) associated with the two absorption peaks at $N_{s}=$ $7.5 \times 10^{10} \mathrm{~cm}^{-2}$ shown in Fig. 2, and the derivative of the ground-state electron density $d n(z) / d z$ (solid lines). The partial agreements between $\delta n(z)$ and $d n(z) / d z$ show that the density fluctuations of each mode correspond to rigid oscillations of the electron slab on each half of the asymmetric well, in analogy to the center-of-mass mode present in perfect parabolic wells.

\section{Figure 4}

Calculated absorption spectra for the asymmetric parabolic quantum well without magnetic

field for various sheet densities $N_{s}$. At high $N_{s}$ the resonances agree with the curvatures of the well, $\omega_{1}=3.41 \mathrm{meV}$ and $\omega_{2}=3.76 \mathrm{meV}$. 